\title{
Effet de différents conditionnements sous vide ou sous atmosphère modifiée sur la conservation de dattes Deglet Nour en Tunisie
}

\author{
Mohsen Achour ${ }^{a \star}$, Sami Ben Amara ${ }^{a}$, Nizar Ben Salem ${ }^{b}$, Asma Jebali $^{c}$, Moktar Hamdi ${ }^{d}$
}

a École Supérieure des Industries Alimentaires de Tunis, 58 av. Alain Savary, Tunis 1003 ,

Tunisie

achour_mohsen@yahoo.fr

${ }^{\mathrm{b}}$ Papparone Ben Yedder, Zone Industrielle Charguia I, Tunis, Tunisie

${ }^{\mathrm{c}}$ Centre Sectoriel de Formation Professionnelle en Industries Alimentaires, Tunis,

Tunisie

d Institut National des Sciences Appliquées et de Technologie, INSAT, Tunis,

Tunisie

* Correspondance et tirés à part

Reçu le 25 novembre 2002 Accepté le 4 février 2003

Fruits, 2003, vol. 58, p. 205-212 (C) 2003 Cirad/EDP Sciences All rights reserved

DOI: $10.1051 /$ fruits:2003008

RESUMEN ESPAÑOL, p. 212

\section{Effect of vacuum and modified atmosphere packaging on Deglet Nour date storage in Tunisia.}

Abstract - Introduction. The aim of our research was to test various techniques of packaging for dates stuffed with almond paste and Deglet Nour natural dates, products of national economic interest in Tunisia. Effects of storage temperatures and packaging types were studied from yeast and mold evolution and water content of stored products. Materials and methods. Natural or stuffed date storage was evaluated by using four packaging types: setting the products in an amorphous polyethylene terephthalate small wrapping (APET) with simple sealing; vacuum packaging or modified atmosphere packaging with injection of two different amounts of a gas mixture $\left(20 \% \mathrm{CO}_{2}\right.$ and $\left.80 \% \mathrm{~N}_{2}\right)$. The sample storage was carried out at three temperatures $\left[(20,30 \text { and } 40)^{\circ} \mathrm{C}\right]$ to cause and accelerate the ageing of the dates and, thus, evaluate their shelf life. Results and discussion. Vacuum and modified atmosphere packaging made it possible to decrease the date dehydration during storage. While being based on the product water content, the stuffed date packaging stored under $20^{\circ} \mathrm{C}$ in small APET wrappings and having received a $10 \%$ gas mixture injection made it possible to obtain a shelf life equal to 6.6 months compared with 4.2 months observed in the case of a simple sealing of these wrappings. For natural dates stored under $20{ }^{\circ} \mathrm{C}$, the application of a partial vacuum packaging increased the shelf life from 3.8 months, obtained for a simple sealing, to 9 months. Conclusion. The use of specific packaging techniques would thus make it possible to preserve for longer the initial stored date quality. The vacuum packaging of natural dates is the most adapted to better protection of the product against yeast and mold proliferation and against dehydration. For stuffed dates, modified atmosphere packaging with a $10 \%$ gas mixture injection proved to be the best for preserving the initial quality of the product during its storage.

Tunisia / Pboenix dactylifera / packaging / controlled atmosphere storage / vacuum packaging / keeping quality / microorganisms

\section{Effet de différents conditionnements sous vide ou sous atmosphère modifiée sur la conservation de dattes Deglet Nour en Tunisie.}

Résumé - Introduction. L'objectif de nos recherches a été de tester différentes techniques de conditionnement de dattes Deglet Nour naturelles et de dattes fourrées à la pâte d'amande, produits d'intérêt économique national en Tunisie. Les effets de la température d'entreposage et du type de conditionnement ont été étudiés par rapport à l'évolution des levures et moisissures et de la teneur en eau des produits stockés. Matériel et méthodes. La conservation des dattes (naturelles ou fourrées) stockées a été évaluée en utilisant quatre modes de conditionnement : mise en barquettes de polyéthylène téréphtalate amorphe (APET) avec simple scellage ; conditionnement sous vide ou emballage sous atmosphère modifiée avec injection de deux différentes doses d'un mélange gazeux $\left(20 \% \mathrm{CO}_{2}\right.$ et $80 \% \mathrm{~N}_{2}$ ). L'entreposage des échantillons a été effectué à trois températures $\left[\left(20,30\right.\right.$ et $\left.40{ }^{\circ}{ }^{\circ} \mathrm{C}\right]$ afin de provoquer et accélérer le vieillissement des dattes et d'évaluer ainsi leurs durées de conservation. Résultats et discussion. Les conditionnements sous atmosphère modifiée et sous vide ont permis de diminuer la déshydratation des dattes durant leur stockage. En se basant sur la teneur en eau des produits, le conditionnement des dattes fourrées stockées à $20^{\circ} \mathrm{C}$ en barquettes d'APET et ayant reçu une injection de $10 \%$ de gaz a permis d'obtenir une durée de conservation égale à 6,6 mois, à comparer avec les 4,2 mois observés dans le cas d'un simple scellage de ces barquettes. Pour les dattes naturelles entreposées à $20^{\circ} \mathrm{C}$, l'application d'un conditionnement sous vide partiel a augmenté la durée de conservation de 3,8 mois (obtenus pour un simple scellage) à 9 mois. Conclusion. L'utilisation de techniques de conditionnement spécifiques permettrait donc de conserver plus longtemps la qualité initiale des dattes stockées. Le conditionnement sous vide des dattes naturelles est le mode le mieux adapté pour une meilleure protection du produit contre le la prolifération des levures et moisissures et contre la déshydratation. Pour les dattes fourrées, le conditionnement sous atmosphère modifiée avec une injection d'un mélange gazeux $\left(20 \% \mathrm{CO}_{2}\right.$ et $\left.80 \% \mathrm{~N}_{2}\right)$ à la dose de $10 \%$ s'est révélé le meilleur pour conserver la qualité initiale du produit durant le stockage.

Tunisie / Pboenix dactylifera / conditionnement / stockage en atmosphère contrôlée / emballage sous vide / aptitude a la conservation / micro-organisme 


\section{Introduction}

L'emballage et le conditionnement des produits alimentaires comportent une grande diversité de formes, de matériaux, de fonctions et de contraintes. Ce secteur, lié aux habitudes alimentaires et aux modes de distribution, est en évolution. Aujourd'hui, l'emballage devient partie intégrante de tout produit alimentaire fini et commercialisé. La qualité du produit, sa conservation, et sa commercialisation sont considérablement influencées par le choix adéquat de l'emballage utilisé. Les interactions contenu-contenant ainsi que les échanges de matière permis par le matériau d'emballage en contact avec le milieu d'entreposage ont fait l'objet de plusieurs travaux de recherche [1]. Les nouvelles techniques de conditionnement et d'emballage ne cessent de se développer afin de mieux conserver le produit emballé et de faciliter son écoulement sur les marchés devenus de plus en plus exigeants.

Le conditionnement sous atmosphère modifiée (Modified Atmosphere Packaging ou AMP) constitue l'une des techniques récentes les plus répandues. Elle a été appliquée sur une gamme diversifiée de produits alimentaires tels que les fruits et légumes [2, 3], les pâtes [4] et les poissons [5]. L'étude de la stabilité des produits alimentaires emballés est souvent réalisée en utilisant la méthode de vieillissement accéléré (Accelerated Shelf Life Testing ou ASLT). Cette approche a été appliquée dans le cas de plusieurs types de produits alimentaires tels que le lait en poudre, le café soluble [6] et les ferments lactiques [7].

Nos travaux ont cherché à tester les techniques de conditionnement sous atmosphère modifiée et sous vide pour des dattes Deglet Nour naturelles et des dattes fourrées à la pâte d'amandes, produits d'intérêt économique national en Tunisie. La conservation de ces produits a été étudiée, en fonction de la température d'entreposage et de la nature du conditionnement, soit dans des barquettes avec simple scellage, soit sous vide partiel ou sous atmosphère modifiée.

\section{Matériel et méthodes}

\subsection{Préparation des dattes}

Des dattes naturelles Deglet Nour non traitées ont été triées en fonction de leur poids en sélectionnant les fruits ayant une masse comprise entre (8 et 9) g et une couleur vive dorée, caractéristique de la variété. Une fois triées, les dattes ont été déposées dans des barquettes en polyéthylène téréphtalate amorphe (APET) avant d'être soumises à différents modes de conditionnement.

Les dattes fourrées ont été préparées à partir de dattes dénoyautées qui ont subi des opérations de fumigation, triage, hydratation et glaçage. La pâte d'amande, préparée au préalable selon un procédé standard, a été façonnée en un rouleau de $1 \mathrm{~cm}$ de diamètre à l'aide d'une extrudeuse, puis découpée en morceaux de $3 \mathrm{~cm}$ de long, utilisés ensuite pour fourrer les dattes dénoyautées.

\subsection{Modes de conditionnement}

Les dattes ainsi préparées ont été placées dans des barquettes en APET, matériau barrière qui convient à la conservation des produits sous différents modes de conditionnement et notamment les conditionnements sous vide et sous atmosphère modifiée [8]. La machine utilisée pour le conditionnement des barquettes est une machine à vide compensé, type Minipack, modèle TSM 95, nécessitant une alimentation manuelle des barquettes. Notre étude a permis de tester les modes de conditionnement suivants :

- conditionnement par un simple scellage des barquettes à l'aide d'un film en APET scellé à $145^{\circ} \mathrm{C}$ pendant $6 \mathrm{~s}$;

- conditionnement sous vide partiel créé au niveau des barquettes (-53000 Pa), suivi d'un scellage thermique par un film en APET :

- conditionnement sous atmosphère modifiée avec une injection à $10 \%$ : un vide est créé au niveau de la barquette, puis une injection d'une quantité de mélange gazeux $\left(20 \% \mathrm{CO}_{2}, 80 \% \mathrm{~N}_{2}\right)$ correspondant à $10 \%$ du volume total de l'enceinte de la machine 
est réalisée, ce qui représente un volume total de gaz injecté de $0,37 \mathrm{~L}$ par barquette. Après injection, les barquettes sont scellées par un film en APET ;

- conditionnement sous atmosphère modifiée avec une injection à $15 \%$; la dose injectée, du même mélange gazeux que précédemment, est alors égale à $15 \%$ du volume total de l'enceinte de la machine, soit un volume total de gaz injecté égal à 0,56 L par barquette.

Les échantillons ont été divisés en trois lots maintenus dans des étuves telles que : lot 1 , température de $20^{\circ} \mathrm{C}$, humidité relative de $50 \%$; lot 2 : température de $30^{\circ} \mathrm{C}$, humidité relative de $41 \%$; lot 3 : température de $40{ }^{\circ} \mathrm{C}$, humidité relative de $31 \%$.

\subsection{Analyses effectuées}

Chacune des analyses effectuées a été répétée trois fois et la moyenne des trois valeurs obtenues a été reportée. La précision préconisée est d'environ $5 \%$.

Deux paramètres aptes à traduire la qualité des produits dattiers stockés ont été considérés :

- Le dénombrement des levures et moisissures a été effectué à partir de trois dilutions à l'aide d'un appareil Stomacher qui permet de réaliser un broyage adéquat et une bonne homogénéisation des échantillons. Le milieu de culture utilisé pour la détermination des levures et moisissures a été le milieu Potato Dextrose Agar, (PDA) acidifié par de l'acide tartrique à $10 \%$ conduisant à un $\mathrm{pH}$ de 3,5 [9]. Les résultats ont été exprimés en "unité formant une colonie " par gramme de produit $\left(\mathrm{UFC} \cdot \mathrm{g}^{-1}\right)$.

- La teneur en eau des produits dattiers a été déterminée par une dessiccation en étuve à $105^{\circ} \mathrm{C}$ jusqu'à poids constant [10]. Un échantillon de $5 \mathrm{~g}$ a été haché, puis il a été étalé sur le fond d'une capsule passée à l'étuve, refroidie dans un dessiccateur et préalablement tarée. Cet échantillon a été séché jusqu'à poids constant dans une étuve réglée à $105^{\circ} \mathrm{C}$ avant de subir luimême un refroidissement dans un dessiccateur et d'être finalement pesé.

\subsection{Principe de la méthode de vieillissement accéléré}

La durée limite de conservation d'un aliment est définie comme étant la période pendant laquelle l'aliment conserve une qualité microbiologique, organoleptique et nutritionnelle acceptable. Selon la nature du produit, cette période peut varier de quelques heures à plusieurs jours ou mois [6]. Par conséquent, la détermination de la durée limite de conservation peut poser parfois des contraintes temporelles. L'utilisation de la méthode du vieillissement accéléré permet alors de pallier ce problème. L'approche est basée sur la détermination expérimentale de la durée de conservation du produit soumis à des conditions sévères d'entreposage. Les résultats obtenus dans de telles conditions peuvent être exploités et extrapolés afin de prédire la durée de conservation du produit s'il était maintenu sous des conditions réelles d'entreposage [11]. Pour cela et afin de préciser un protocole expérimental, nous avons suivi la variation des propriétés du produit liées à la dégradation de sa qualité durant l'entreposage. Chaque propriété choisie a constitué un indice de qualité. En modélisant la cinétique apparente de dégradation de la qualité du produit au travers des indices choisis, il a été possible d'établir des modèles de prédiction de la variation des indices en fonction du temps et de la température d'entreposage. Par exemple, en supposant que ces réactions d'altération sont d'ordre 0 ou 1 , on a défini les modèles suivants:

$$
\begin{array}{cc}
{[\mathrm{A}]=[\mathrm{A}]_{0} \pm \mathrm{k} t} & (\text { ordre }=0) \\
{[\mathrm{A}]=[\mathrm{A}]_{0} \exp ( \pm \mathrm{k} t)} & (\text { ordre }=1)
\end{array}
$$

dans lesquels [A] est la valeur de l'indice de qualité à l'instant $t$, [A $]_{0}$ est la valeur initiale de l'indice de qualité à $t=0$, k est la constante de la réaction apparente de dégradation, $t$ représente le temps d'entreposage.

En supposant que l'effet de la température sur la cinétique apparente des réactions d'altération suit la loi exponentielle 


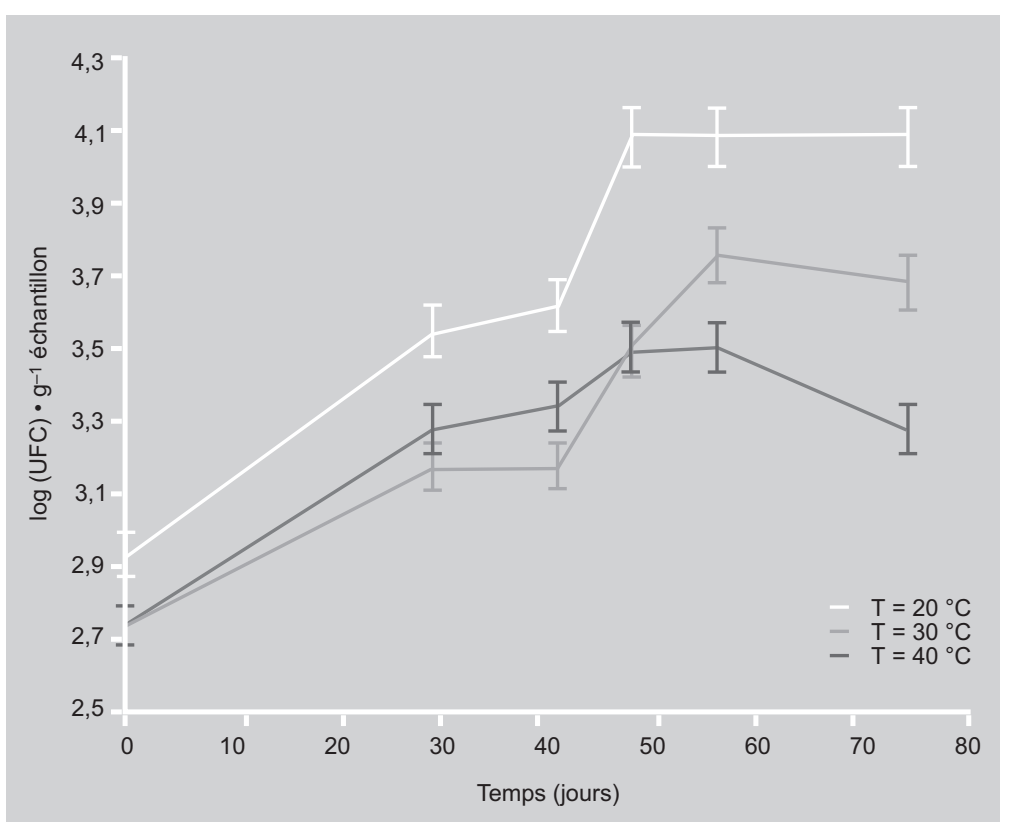

Figure 1.

Effet de la température sur l'évolution des levures et moisissures sur des dattes naturelles du type Deglet Nour stockées sous atmosphère modifiée, ayant subi, après application d'un vide, une injection d'une quantité de mélange gazeux (20\% $\mathrm{CO}_{2}, 80 \% \mathrm{~N}_{2}$ ) correspondant à $15 \%$ du volume total de l'enceinte utilisée pour le conditionnement (UFC : unité formant une colonie).

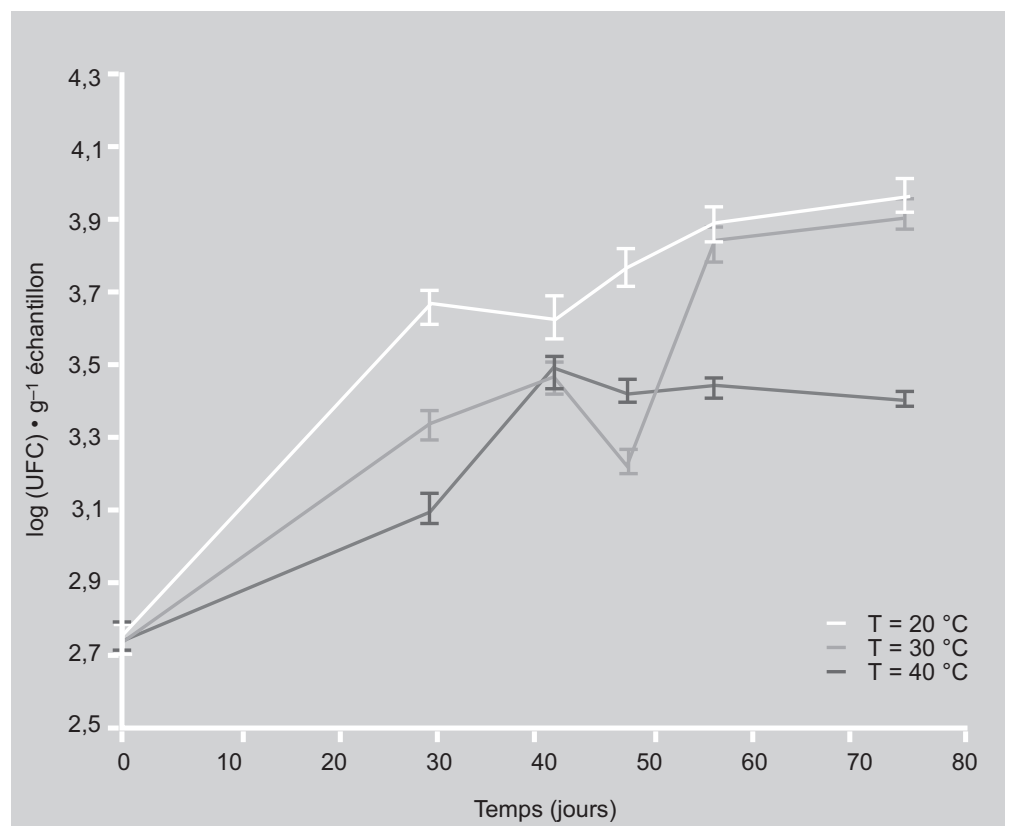

Figure 2.

Effet de la température sur l'évolution des levures et moisissures sur des dattes Deglet Nour fourrées à la pâte d'amande et conditionnées par simple scellage des barquettes, à $145^{\circ} \mathrm{C}$ pendant $6 \mathrm{~s}$, à l'aide d'un film en polyéthylène téréphtalate amorphe (UFC : unité formant une colonie).
d'Arrhenius, les modèles de prédiction de [A] deviennent :

$$
[\mathrm{A}]=[\mathrm{A}]_{0} \pm \mathrm{k}_{0} t \exp \left(-\mathrm{E}_{\mathrm{a}} / \mathrm{RT}\right) \quad(\text { ordre }=0)
$$

$[\mathrm{A}]=[\mathrm{A}]_{0} \exp \left[ \pm \mathrm{k}_{0} \mathrm{t} \exp \left(-\mathrm{E}_{\mathrm{a}} / \mathrm{RT}\right)\right]($ ordre $=1)$

dans lesquels $\mathrm{k}_{0}$ est le terme pré-exponentiel, $\mathrm{E}_{\mathrm{a}}$ est l'énergie d'activation, $\mathrm{R}$ est la constante des gaz parfaits.

Le suivi expérimental de la variation de l'indice de qualité en fonction du temps pour différentes températures d'entreposage permet de déterminer les constantes $\mathrm{k}_{0}$ et $\mathrm{E}_{\mathrm{a}}$ et ainsi d'établir les modèles de prédiction de [A] pour un entreposage donné. Ces modèles peuvent être convertis en modèles de prédiction de la durée limite de conservation si une valeur "seuil" de l'indice de qualité au-delà de laquelle le produit est jugé inacceptable pour la consommation est fixée. Cette valeur pourrait être déterminée par la législation ou par les exigences du marché ou du consommateur.

\section{Résultats et discussion}

\section{1. Évolution des levures et moisissures}

La croissance des levures et des moisissures sur dattes naturelles (figure 1) ou sur dattes fourrées (figure 2) a été plus importante sous températures d'entreposage de (20 ou 30) ${ }^{\circ} \mathrm{C}$ que sous $40{ }^{\circ} \mathrm{C}$. Cette observation est à relier au type d'altérations microbiennes développées dans l'un et l'autre cas : moisissures du groupe Aspergillus glarcus et Aspergillus chevalier, principaux agents d'altération de la pâte d'amandes, et moisissures du groupe Aspergillus niger susceptibles d'altérer les dattes entières [9]. Ces différents groupes de moisissures se développent à des températures allant de (15 à 30) ${ }^{\circ} \mathrm{C}$. En revanche, à $40{ }^{\circ} \mathrm{C}$, le phénomène de déshydratation est accéléré et la teneur en eau des dattes fourrées décroît rapidement ; par suite, l'activité de l'eau du produit diminue, ce qui ne permet pas le développement des levures et moisissures qui nécessitent une activité d'eau supérieure à 0,75 . 
Lorsque l'évolution des levures et moisissures a été étudiée en fonction des différents modes de conditionnement, les dattes fourrées et emballées sous simple scellage ont toujours présenté la croissance la plus rapide, quelle que soit la température d'entreposage considérée (figures 3, 4).

En général, le conditionnement sous atmosphère modifiée a permis une bonne conservation des produits alimentaires en raison de la présence de $\mathrm{CO}_{2}$ qui est un agent bactériostatique et fongistatique retardant la croissance et réduisant la vitesse de multiplication des moisissures et des bactéries aérobies. La différence entre l'effet du conditionnement sous atmosphère modifiée des dattes fourrées, avec une injection de gaz à $10 \%$ ou avec une injection à $15 \%$, n'a pas été clairement perceptible sur l'évolution des levures et des moisissures (figure 3) ; cela pourrait être expliqué par le choix non optimal du volume et de la composition du gaz injecté utilisé : alors que le $\mathrm{CO}_{2}$ ne serait efficace qu'à des teneurs supérieures à $20 \%$, nous avons utilisé un mélange gazeux à $20 \%$ de $\mathrm{CO}_{2}$ et $80 \%$ de $\mathrm{N}_{2}$, commercialisé sous le nom de Aligal 12 qui est l'Aligal le plus adapté au conditionnement de la pâte d'amande seule.

Quelle qu'ait été la température d'entreposage, l'emballage sous vide a permis d'observer la croissance la plus lente de levures et de moisissures. Le conditionnement sous vide a permis de priver les micro-organismes aérobies de l'oxygène qui était présent dans les barquettes avant le conditionnement et, par conséquent,

\section{Figure 4.}

Effet de différents types de conditionnement sur l'évolution de levures et moisissures sur des dattes Deglet Nour fourrées à la pâte d'amande et stockées à $30^{\circ} \mathrm{C}$ (UFC : unité formant une colonie). Simple scellage : scellage à $145^{\circ} \mathrm{C}$ pendant $6 \mathrm{~s}$, à l'aide d'un film en polyéthylène téréphtalate amorphe (APET); $15 \%$ MAP : stockage sous atmosphère modifiée, avec injection d'une quantité de mélange gazeux $\left(20 \% \mathrm{CO}_{2}, 80 \% \mathrm{~N}_{2}\right)$ correspondant à $15 \%$ du volume total de l'enceinte utilisée pour le conditionnement ; $10 \%$ MAP : idem $15 \%$ MAP mais injection de $10 \%$ du mélange gazeux seulement ; vide : vide partiel créé dans les barquettes suivi d'un scellage thermique par un film en APET.

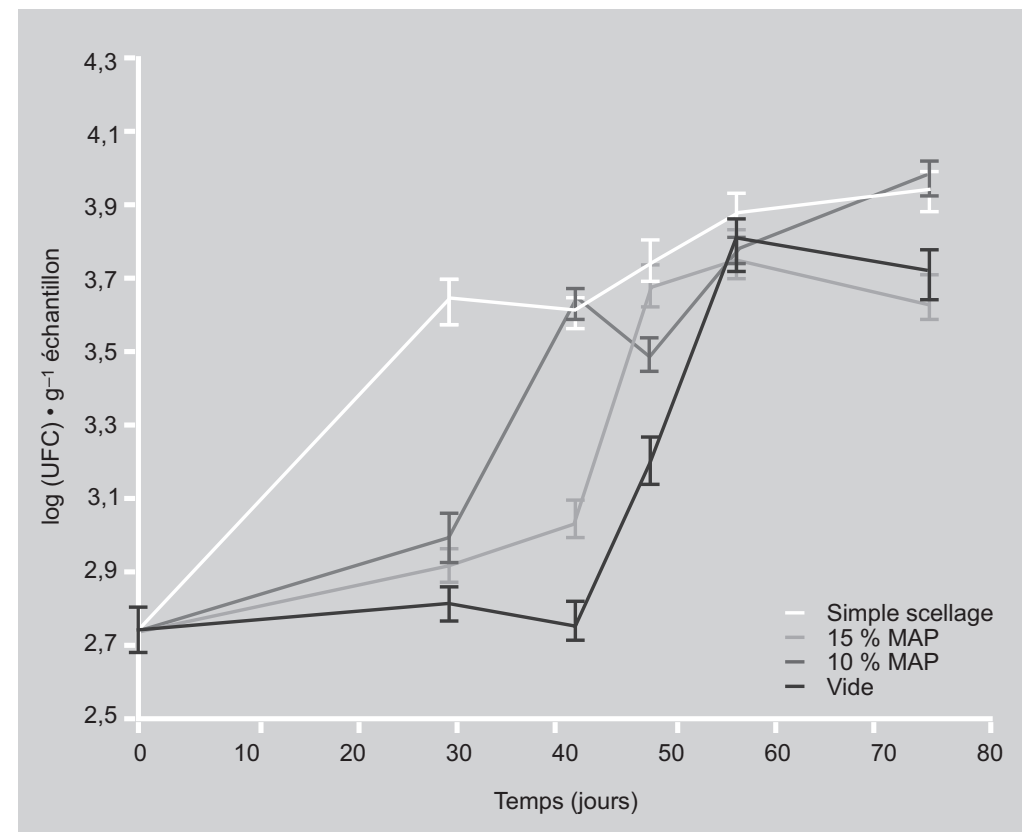

\section{Figure 3.}

Effet de différents types de conditionnement sur l'évolution de levures et moisissures sur des dattes Deglet Nour fourrées à la pâte d'amande et stockées à $20^{\circ} \mathrm{C}$ (UFC : unité formant une colonie). Simple scellage : scellage à $145^{\circ} \mathrm{C}$ pendant $6 \mathrm{~s}$, à l'aide d'un film en polyéthylène téréphtalate amorphe (APET); $15 \%$ MAP : stockage sous atmosphère modifiée, avec injection d'une quantité de mélange gazeux $\left(20 \% \mathrm{CO}_{2}, 80 \% \mathrm{~N}_{2}\right)$ correspondant à $15 \%$ du volume total de l'enceinte utilisée pour le conditionnement ; $10 \%$ MAP : idem $15 \%$ MAP mais injection de $10 \%$ du mélange gazeux seulement ; vide : vide partiel créé dans les barquettes suivi d'un scellage thermique par un film en APET.

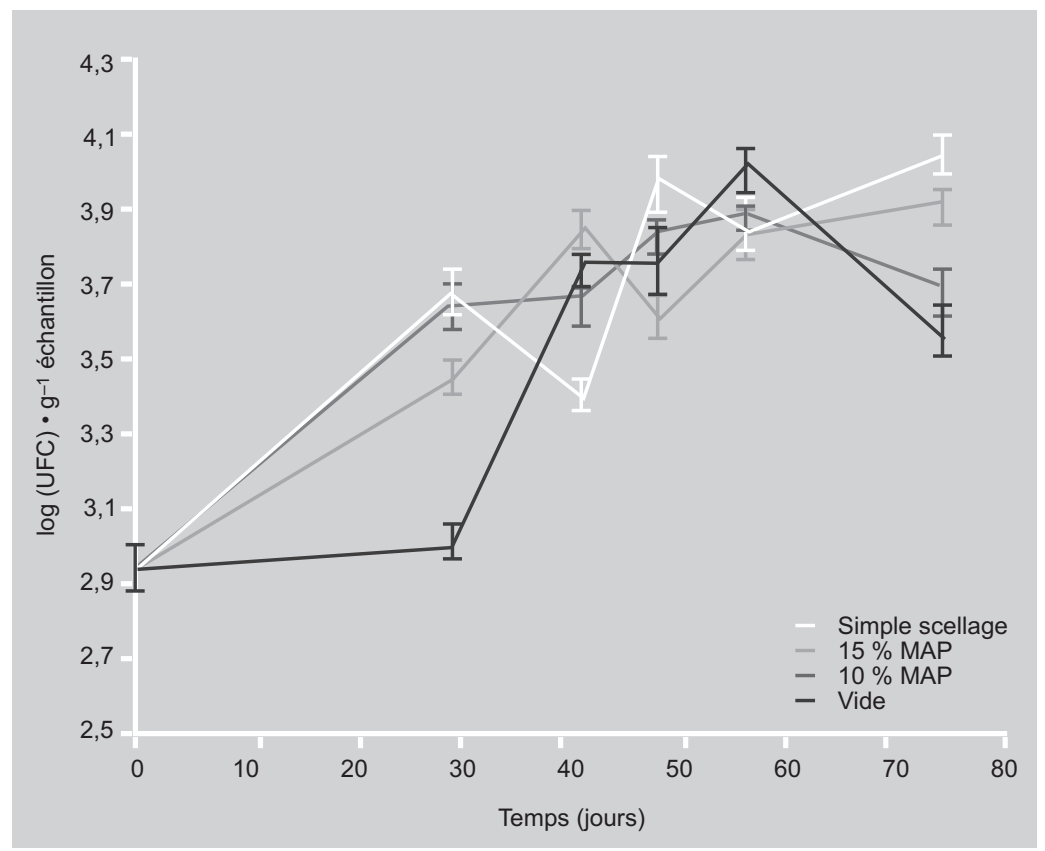


leur croissance a été ralentie. Ce type d'emballage serait donc à recommander pour préserver au mieux les dattes four-

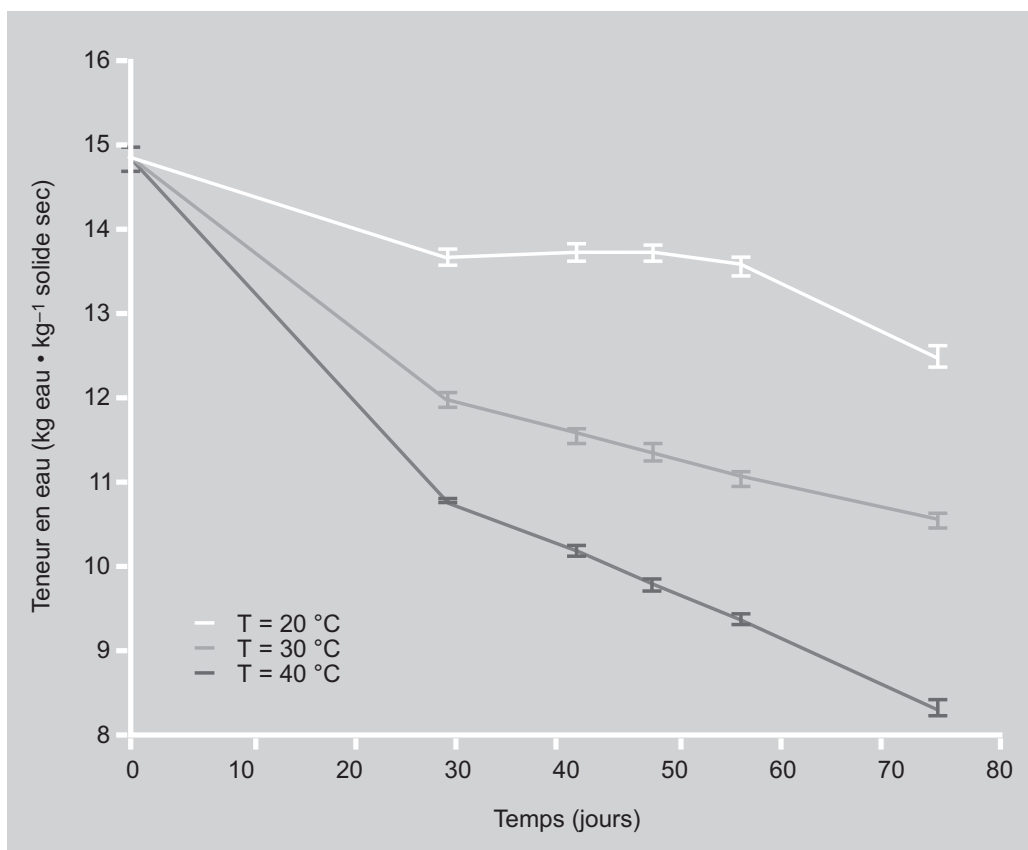

Figure 5

Effet de la température sur l'évolution de la teneur en eau de dattes naturelles du type Deglet Nour fourrées à la pâte d'amande et conditionnées par simple scellage des barquettes, à $145^{\circ} \mathrm{C}$ pendant $6 \mathrm{~s}$, à l'aide d'un film en polyéthylène téréphtalate amorphe.

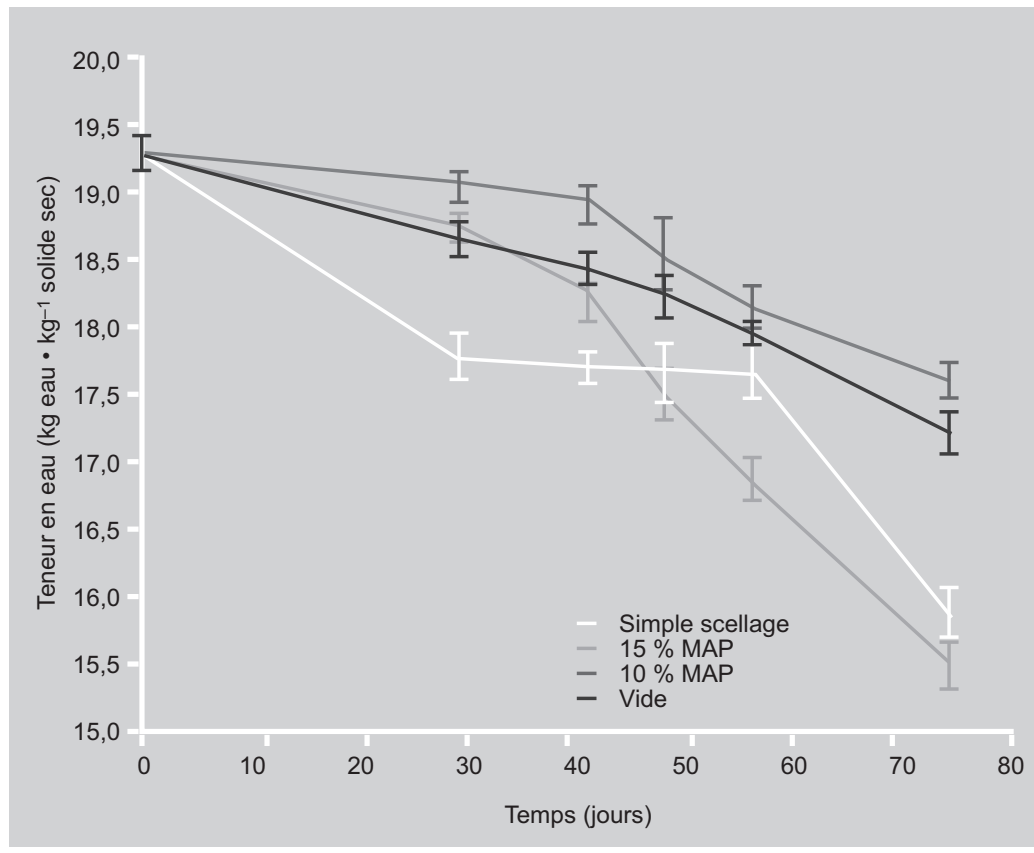

rées contre les altérations causées par les levures et les moisissures.

\section{2. Évolution de la teneur en eau}

La teneur en eau des dattes affecte considérablement leur qualité sensorielle. Cette teneur est fonction de l'humidité relative du milieu ambiant et de la température d'entreposage. Cette relation est décrite par les isothermes de sorption qui ont été établis dans le cas des dattes naturelles de type Deglet Nour [12]. Au cours de nos expérimentations, dans le cas des dattes fourrées conditionnées sous simple scellage, quel qu'ait été la température utilisée pour stocker les dattes fourrées, la perte d'eau a été importante malgré l'effet barrière à la vapeur d'eau assuré par l'APET enveloppant les barquettes (figure 5). Cette perte serait due à l'existence d'un écart de pression de vapeur d'eau de par et d'autre de la paroi de la barquette. Ce gradient serait d'autant plus important que la température d'entreposage augmente (figure 5).

L'étude de l'effet du type de conditionnement sur l'évolution de la teneur en eau des dattes a montré que les dattes naturelles entreposées à $20^{\circ} \mathrm{C}$ et conditionnées sous simple scellage présentaient une diminution de leur teneur en eau relativement plus importante que celle des lots conditionnés sous vide ou sous atmosphère modifiée (figure ๑).

\subsection{Estimation de la durée de conservation}

Dans le cadre de notre travail, la détermination des durées optimales de conservation

\footnotetext{
Figure 6.

Effet de différents types de conditionnement sur l'évolution de la teneur en eau de dattes naturelles du Deglet Nour stockées à $20^{\circ} \mathrm{C}$. Simple scellage : scellage à $145^{\circ} \mathrm{C}$ pendant $6 \mathrm{~s}$, à l'aide d'un film en polyéthylène téréphtalate amorphe (APET); $15 \%$ MAP : stockage sous atmosphère modifiée, avec injection d'une quantité de mélange gazeux (20\% $\mathrm{CO}_{2}, 80 \% \mathrm{~N}_{2}$ ) correspondant à $15 \%$ du volume total de l'enceinte utilisée pour le conditionnement ; $10 \%$ MAP : idem $15 \%$ MAP mais injection de $10 \%$ du mélange gazeux seulement ; vide : vide partiel créé dans les barquettes suivi d'un scellage thermique par un film en APET.
} 
Tableau I.

Estimation de la durée de conservation (en jours) de dattes Deglet Nour fourrées à la pâte d'amande, basée sur un seuil de teneur en eau de cette pâte égal à 4,5 \%.

\begin{tabular}{lcccc} 
Température $\left({ }^{\circ} \mathrm{C}\right)$ & Simple scellage & Vide & MAP à $10 \%$ & MAP à $15 \%$ \\
\hline 20 & 127 & 133 & 198 & 139 \\
30 & 78 & 84 & 110 & 92 \\
40 & 49 & 55 & 63 & 63
\end{tabular}

MAP : Modified Atmosphere Packaging ou conditionnement sous atmosphère modifiée.

\section{Tableau II.}

Estimation de la durée de conservation (en jours) de dattes naturelles de type Deglet Nour, basée sur un seuil de teneur en eau des dattes égal à $18 \%$.

\begin{tabular}{lcccc} 
Température $\left({ }^{\circ} \mathrm{C}\right)$ & Simple scellage & Vide & MAP à $10 \%$ & MAP à $15 \%$ \\
\hline 20 & 114 & 272 & 151 & 215 \\
30 & 36 & 59 & 47 & 60 \\
40 & 12 & 14 & 16 & 18
\end{tabular}

MAP : Modified Atmosphere Packaging ou conditionnement sous atmosphère modifiée.

des dattes fourrées et des dattes naturelles a été basée sur la teneur en eau considérée comme l'indice de qualité le plus significatif pour évaluer la qualité microbiologique ou sensorielle des produits.

Pour les dattes fourrées, une teneur en eau de la pâte d'amande inférieure à 4,5\% correspond à une qualité organoleptique inacceptable du produit du fait d'un dessèchement pouvant fragiliser la pâte. Par ailleurs, pour une teneur en eau inférieure à $18 \%$, les dattes naturelles de type Deglet Nour perdent leur qualité organoleptique habituelle et sont déclassées. La durée de conservation pourrait donc être considérée dans ce cas comme une date limite d'utilisation optimale. En appliquant les notions décrites précédemment de la cinétique apparente de dégradation de la qualité des dattes, l'analyse a montré que, dans le cas des dattes fourrées et naturelles, la réaction apparente de la dégradation de la qualité (diminution de la teneur en eau) était d'ordre 1. Sur la base des seuils de teneur en eau choisis, les durées optimales de conservation des dattes fourrées et naturelles ont été déterminées (tableaux I, II). Nos résultats ont confirmé que la température avait un effet négatif sur la conservation de la qualité des dattes et que les conditionnements sous vide et sous atmosphère modifiée constituaient les modes les mieux adaptés à la conservation des dattes vis-àvis des problèmes de déshydratation. L'application de la loi d'Arrhenius a permis d'estimer des énergies d'activation comprises entre $(-62700$ et 83600$) \mathrm{J} \cdot \mathrm{mol}^{-1}$, ce qui indique une sensibilité moyenne de la réaction apparente de dégradation de la qualité vis-à-vis de la température d'entreposage [13].

\section{Conclusions}

Les résultats obtenus à l'issue de nos expérimentations montrent que le conditionnement sous vide des dattes naturelles est le mode le mieux adapté pour une meilleure protection des fruits stockés contre le la prolifération des levures et moisissures et contre le phénomène de déshydratation. Pour les dattes fourrées, le conditionnement sous atmosphère modifiée avec une injection d'un mélange gazeux (20\% $\mathrm{CO}_{2}$ et $\left.80 \% \mathrm{~N}_{2}\right)$ à la dose de $10 \%$ s'est révélé le meilleur pour conserver la qualité initiale du produit durant son stockage. 


\section{Références}

[1] Bureau G., Multon J.L., L'emballage des denrées de grande consommation, Technique et documentation, Tec. \& Doc. Lavoisier, Paris, France, 1989.

[2] Kader A.A., Zagory D., Kerbel E.L., Modified atmosphere packaging of fruits and vegetables, Crit. Rev. Food Sci. 28 (1) (1989) 1-30.

[3] Hong J.H., Gross K.C., Maintaining quality of fresh-cut tomato slices through modified atmosphere packaging and low temperature storage, J. Food Sci. 66 (7) (2001) 960-965.

[4] Besson P., Le conditionnement sous atmosphère modifiée, Doss. Sci. Inst. Fr. Nutr. 3 (1993) 39-55.

[5] Parkin K.L., Brown W.D., Preservation of seafood with modified atmospheres, in: Chemistry and biochemistry of marine food products, Martin R.E., Flick G.J., Hebard C.E., Ward D.R. (Eds.), AVI Publ. Co., Westport, CT, USA, 1982, pp. 453-465.

[6] Labuza T.P., Shelf-life testing of foods, in: Proc. 2nd Saudi Arabian Conference on Food Science and Nutrition, Saudi Arabia, 1984.
[7] Achour M., Mtimet N., Cornelius C., Zgouli S., Mahjoub A., Thonart P., Hamdi M., Application of the accelerated shelf life testing method (ASLT) to study the survival rates of freeze-dried Lactococcus starter cultures, $\mathrm{J}$. Chem. Technol. Biot. 76 (6) (2001) 624-628.

[8] Chomon P., L'emballage souple dans l'agroalimentaire, Emball. Mag., 1992, Paris, France.

[9] Bourgeois C.M., Microbiologie alimentaire : aspect microbiologique de la sécurité et de la qualité alimentaire, tome 1, Tec. \& Doc. Lavoisier, Paris, France, 1988.

[10] Cheftel J.C., Cheftel H., Introduction à la biochimie et la technologie des aliments, Vol. 1 , Tec. \& Doc. Lavoisier, Paris, France, 1976.

[11] Labuza T.P., Schmidl M.K., Accelerated shelf-life testing of foods, Food Technol.Chicago 39 (9) (1985) 57-64.

[12] Achour M., Hamdi S., Benzarti L., Détermination des isothermes de sorption de dattes Deglet Nour entreposées en Tunisie, Fruits 54 (2) (1999) 123-128.

[13] Levenspiel O., Chemical reaction engineering, 2nd edition, John Wiley \& Sons, New York, USA, 1972

\section{Efecto de distintos acondicionamientos al vacío o con atmósfera modificada en la conservación de dátiles Deglet Nour en Túnez.}

Resumen - Introducción. El objetivo de nuestras investigaciones consistió en probar distintas técnicas de acondicionamiento de dátiles Deglet Nour naturales y de dátiles rellenos con pasta de almendra, productos de interés económico nacional en Túnez. Se estudiaron los efectos de la temperatura de almacenamiento y del tipo de acondicionamiento con relación a la evolución de las levaduras y mohos y del contenido de agua de los productos almacenados. Material y métodos. Se evaluó la conservación de los dátiles (naturales o rellenos) almacenados utilizando cuatro métodos de acondicionamiento: colocación en bandejas de polietilentereftalato amorfo (APET) con simple sellado, acondicionamiento al vacío o embalaje en atmósfera modificada con inyección de dos dosis diferentes de una mezcla gaseosa $\left(20 \% \mathrm{CO}_{2}\right.$ y $\left.80 \% \mathrm{~N}_{2}\right)$. El almacenamiento de las muestras se efectuó con tres temperaturas $\left[(20,30 \mathrm{y} 40)^{\circ} \mathrm{C}\right]$ para provocar y acelerar el envejecimiento de los dátiles y poder evaluar las duraciones de conservación. Resultados y discusión. Los acondicionamientos en atmósfera modificada y al vacío permitieron disminuir la deshidratación de los dátiles durante su almacenamiento. Tomando como base el contenido de agua de los productos, el acondicionamiento de los dátiles rellenos almacenados a $20^{\circ} \mathrm{C}$ en bandejas de APET y con una inyección del 10\% de gas permitió lograr una duración de conservación de 6,6 meses frente a los 4,2 meses observados en el caso de un simple sellado de estas bandejas. En los dátiles naturales almacenados a $20{ }^{\circ} \mathrm{C}$, la aplicación de un acondicionamiento al vacío parcial aumentó la duración de conservación de 3,8 meses (obtenidos con simple sellado) a 9 meses. Conclusión. La utilización de técnicas de acondicionamiento específicas permitiría conservar mucho más tiempo la calidad inicial de los dátiles almacenados. El acondicionamiento al vacío de los dátiles naturales es el método mejor adaptado para una mejor protección del producto contra la proliferación de levaduras y mohos y contra la deshidratación. Para los dátiles rellenos, el acondicionamiento en atmósfera modificada con una inyección de una mezcla gaseosa $\left(20 \% \mathrm{CO}_{2}\right.$ y $\left.80 \% \mathrm{~N}_{2}\right)$ a una dosis del $10 \%$ produjo los mejores resultados para conservar la calidad inicial del producto durante el almacenamiento.

Túnez / Pboenix dactylifera / empaquetado / almacenamiento atmósfera controlada / embalaje en vacío / aptitud para la conservación / microorganismos 